\title{
LXIII. The symmetrical vibrations of conducting surfaces of revolution
}

\author{
J.W. Nicholson B.A. M.Sc.
}

To cite this article: J.W. Nicholson B.A. M.Sc. (1906) LXIII. The symmetrical vibrations of conducting surfaces of revolution, Philosophical Magazine Series 6, 11:65, 703-721, DOI: $10.1080 / 14786440609463488$

To link to this article: http://dx.doi.org/10.1080/14786440609463488

曲 Published online: 16 Apr 2009.

Submit your article to this journal $[\pi$

Џ Article views: 2

Q View related articles $\square$

Citing articles: 1 View citing articles $\square$ 
accurate, and were there no phase change. On account of the phase change as determined from the cadmium observations, the fractional parts should be those entered in the 7th column. The differences are trifling, except in the cases of 5016 and 4713 . The proportional corrections by which the $\lambda$ 's of column 5 are to be increased are set out in column 8 expressed in millionths ; but of course an accuracy of 4 or 5 millionths is hardly to be expected in results from a single set of observations with the $1 \mathrm{~mm}$. gauge.

In the observations (Table II.) with the $5 \mathrm{~mm}$. gauge the comparisons were with the cadmium green, for which $\mathrm{P}$ is assumed to be 18753.9. , corresponding to 14814.00 for cadmium red. The numbers given embody the results of three days' observations, but they do not include the wavelengths 4713,4472 . The procedure is the same as for Table I. If the observations with the $5 \mathrm{~mm}$. gauge stood alone, we should be in doubt whether $\mathrm{P}$ for 5016 should be 19016.95 , or 19015.95 . The results with the $1 \mathrm{~mm}$. gauge show that the latter alternative must be chosen. Except in this respect, the $5 \mathrm{~mm}$. results are independent; and they are of course to be preferred as presumably more accurate. The final numbers for helium are therefore those given in column 6 of Table II.

The only further remark that I will make is that the observations on the helium yollow (5876) are not improbably somewhat embarrassed by a companion of feeble luminosity which could not be separated. In the $5 \mathrm{~mm}$. apparatus the two components would be nearly but not quite in coincidence.

LXIII. The Symmetrical Vibrations of Conducting Surfaces of Revolution. By J. W. Nicholson, B.A., M.Sc., Scholar of Trinity College, and Isaac Newton Student in the University of Cambridge*.

T $\mathrm{N}$ a previous paper $\dagger$, the author investigated the possible electrical periods of the space between two perfectly conducting elliptic cylinders belonging to a confocal system. The vibrations of symmetrical type were alone considered. The object of the present paper is to obtain a list of the simpler surfaces of revolution, including cylinders as a particular case, whose vibrations admit readily of analytical treatment, and to examine certain important cases in detail.

* Communicated by the Author.

+ Phil. Mag. Aug. 1905. 
The entire investigation is confined to symmetrical vibrations, which are the most interesting from a physical point of view.

The electric and magnetic vectors are first expressed in a convenient manner in terms of two potential functions satisfying a certain differential equation.

Let any point of space be defined by (1) cylindrical coordinates $(\rho \omega z)$, where $z$ is the distance along an axis, $\rho$ the perpendicular upon that axis, and $\omega$ the longitude of the meridian of the point; and (2) coordinates $(\alpha \beta \omega)$, where

$$
\rho+\iota z=f(\alpha+\iota \beta) \text {. . . . . . }
$$

Then the surfaces $\alpha=$ const., $\beta=$ const., $\omega=$ const., form three mutually orthogonal systems. The last is a system of planes, and the others are surfaces of revolution about $z$.

The space elements normal to the three surfaces, one of each system, through any point, are

$$
d n_{1}=\frac{d \alpha}{p_{1}}, \quad d n_{2}=\frac{d \beta}{p_{2}}, \quad d n_{3}=\frac{d \omega}{p_{3}} . . .
$$

As usual $(x y z),(a b c),(u v w)$ will denote components of electric force, magnetic induction, and current, but measured along the directions whose cosines are

$$
\left(d n_{1}, d n_{2}, d n_{3}\right) /\left(d n_{1}{ }^{2}+d n_{2}{ }^{2}+d n_{3}{ }^{2}\right)^{\frac{1}{2}} .
$$

The magnetic force is $(a, b, c) / \mu$, if $\mu$ be the permeability. For all investigations here contemplated, $\mu=1$.

By the circuital relation expressing Faraday's law, since $\frac{\partial}{\partial \omega}=0$ for symmetrical vibrations,

$$
\begin{aligned}
\frac{-\dot{a}}{p_{2} p_{\dot{b}}} & =\frac{\partial}{\partial \beta} \cdot \frac{\tilde{z}}{p_{3}}, \quad \frac{\dot{b}}{p_{1} p_{3}}=\frac{\partial}{\partial \alpha} \cdot \frac{\tilde{z}}{p_{3}}, \\
& \frac{-\dot{c}}{p_{1} p_{2}}=\frac{\partial}{\partial \alpha} \cdot \frac{\gamma}{p_{2}}-\frac{\partial}{\partial \beta} \cdot \frac{x}{p_{1}}, \quad . \quad . \quad . \quad .
\end{aligned}
$$

where dots denote differentiations with respect to $t$.

The appropriate expression of Ampère's law leads to

$$
\begin{array}{r}
\frac{\dot{x}}{\mathrm{~V}^{2} p_{2} p_{3}}=\frac{\partial}{\partial \beta} \cdot \frac{c}{p_{3}}, \quad \frac{\dot{y}}{\mathrm{~V}^{2} p_{1} p_{3}}=-\frac{\partial}{\partial \alpha} \cdot \frac{c}{p_{3}}, \\
\frac{-\dot{z}}{\mathrm{~V}^{2} p_{1} p_{2}}=\frac{\partial}{\partial \alpha} \cdot \frac{b}{p_{2}}-\frac{\partial}{\partial \beta} \cdot \frac{a}{p_{1}}, . . .
\end{array}
$$

writing $4 \pi \mathrm{V}^{2}(u, v, w)=(x, y, z)$, where $\mathrm{V}$ is the velocity of propagation of electromagnetic disturbances in the medium between the conductors. 
Vibrations of Conducting Surfaces of Revolution.

In order that these equations should be mutually compatible, the solenoidal conditions

$$
\begin{aligned}
& \frac{\partial}{\partial \alpha} \cdot \frac{x}{p_{2} p_{3}}+\frac{\partial}{\partial \beta} \cdot \frac{y}{p_{1} p_{3}}=0 \\
& \frac{\partial}{\partial \alpha} \cdot \frac{a}{p_{2} p_{3}}+\frac{\partial}{\partial \beta} \cdot \frac{b}{p_{1} p_{3}}=0
\end{aligned}
$$

must be satisfied, again writing $\frac{\partial}{\partial \omega}=0$.

Thus if $\phi$ [and $\psi$ are two functions of $\alpha$ and $\beta$, we may write

$$
\begin{array}{ll}
x=p_{2} p_{3} \frac{\partial \phi}{\partial \beta}, & y=-p_{1} p_{3} \frac{\partial \phi}{\partial \alpha}, \\
a=p_{2} p_{3} \frac{\partial \psi}{\partial \beta}, & b=-p_{1} p_{3} \frac{\partial \psi}{\partial \alpha}, \quad . \quad .
\end{array}
$$

whence by (3),

$$
\frac{\dot{c}}{p_{1} p_{2}}=\frac{\partial}{\partial \alpha} \cdot\left(\frac{p_{1} p_{3}}{p_{2}} \frac{\partial \phi}{\partial \alpha}\right)+\frac{\partial}{\partial \beta}\left(\frac{p_{2} p_{3}}{p_{1}} \frac{\partial \phi}{\partial \beta}\right) .
$$

Thus with (4), writing $\frac{1}{\mathrm{~V}^{2}} \frac{\partial^{2}}{\partial t^{2}}=-k^{2}$, where $\frac{2 \pi}{k}$ is the
ave-length,

$$
\frac{\partial}{\partial \beta} \frac{p_{1} p_{2}}{p_{3}}\left\{\frac{\partial}{\partial \alpha}\left(\frac{p_{1} p_{3}}{p_{2}} \frac{\partial \phi}{\partial \alpha}\right)+\frac{\partial}{\partial \beta}\left(\frac{p_{2} p_{3}}{p_{1}} \frac{\partial \phi}{\partial \beta}\right)\right\}+k^{2} \frac{\partial \phi}{\partial \beta}=0 .
$$

Similarly from (3) and (4)

$$
\frac{\partial}{\partial \alpha} \frac{p_{1} p_{2}}{p_{3}}\left\{\frac{\partial}{\partial \alpha}\left(p_{1} p_{3} \frac{\partial \phi}{\partial \alpha}\right)+\frac{\partial}{\partial \beta}\left(\frac{p_{2} p_{3}}{p_{1}} \frac{\partial \phi}{\partial \beta}\right)\right\}+k^{2} \frac{\partial \phi}{\partial \alpha}=0 \text {. }
$$

Thus

$$
\frac{\partial}{\partial \alpha}\left(\frac{p_{1} p_{3}}{p_{2}} \frac{\partial \phi}{\partial \alpha}\right)+\frac{\partial}{\partial \beta}\left(\frac{p_{2} p_{3}}{p_{1}} \frac{\partial \phi}{\partial \beta}\right)+\frac{k^{2} p_{3}}{p_{1} p_{2}} \phi=0 . .
$$

By the symmetry of the relations, $\psi$ must satisfy the same characteristic equation.

The independence of the functions $\phi$ and $\psi$, and of the corresponding components of force $(x, y, c),(a, b, z)$ derived therefrom, allows of the extension to surfaces of revolution of a result noticed by Macdonald * for the case of spheres. The result so extended may be stated:-

The most general symmetrical oscillation possible in the space between two surfaces of revolution belonging to one of three orthogonal systems of surfaces, is an additive combination of two types: (1) a type in which the components

* Electric Waves, Chap. 3. 
of electric force at any point normal to two of the surfaces through that point are zero; and (2) a type in which the same property is true of the magnetic force. As a particular case, one of the surfaces of revolution may be absent.

The components of force are, in the most general oscillation,

$$
\begin{array}{ll}
x=p_{2} p_{3} \frac{\partial \phi}{\partial \beta}, & y=-p_{1} p_{3} \frac{\partial \phi}{\partial \alpha}, \\
a=p_{2} p_{3} \frac{\partial \psi}{\partial \beta}, & l=-p_{1} p_{3} \frac{\partial \psi}{\partial \alpha}, \\
z=\frac{-p_{3}}{\mathrm{~V}^{2}} \frac{\partial \psi}{\partial t}, & e=\frac{-p_{3}}{\mathrm{~V}^{2}} \frac{\partial \phi}{\partial t} \text { by }(3) \text { io }(6) .
\end{array}
$$

Now the element of arc in any direction $\lambda$ is

$$
d s=\left\{\left(\frac{\partial x}{\partial \lambda}\right)^{2}+\left(\frac{\partial y}{\partial \lambda}\right)^{2}+\left(\frac{\partial z}{\partial \lambda}\right)^{2}\right\}^{\frac{1}{2}} d \lambda .
$$

Taking $\lambda=\alpha, \beta, \omega$ successively,

$$
d n_{1}=\left\{\left(\frac{\partial \rho}{\partial \alpha}\right)^{2}+\left(\frac{\partial z}{\partial \alpha}\right)^{2}\right\}^{\frac{1}{3}} d \alpha=\sqrt{\frac{\partial(\rho, z)}{\partial(\alpha, \beta)}} d \alpha,
$$

by a property of conjugate functions,

similarly

$$
d n_{2}=\sqrt{\frac{\partial(\rho, z)}{\partial(\alpha, \beta)}} d \beta
$$

Thus

$$
d n_{3}=\rho d \omega .
$$

$$
\frac{1}{p_{1}^{2}}=\frac{1}{p_{2}^{2}}=\frac{\partial(\rho, z)}{\partial(\alpha, \beta)} \text { and } p_{3}=\frac{1}{\rho}, \ldots .
$$

and (7) becomes

$$
\frac{\partial^{2} \phi}{\partial \alpha^{2}}+\frac{\partial^{2} \phi}{\partial \beta^{2}}+\frac{1}{\rho}\left(\frac{\partial \phi}{\partial \alpha} \frac{\partial \rho}{\partial \alpha}+\frac{\partial \phi}{\partial \beta} \frac{\partial \rho}{\partial \beta}\right)+k^{2} \phi \frac{\partial(\rho, z)}{\partial(\alpha, \beta)}=0 \text {. }
$$

In order to obtain the electrical periods between two perfectly conducting surfaces of the family, it is necessary to find solutions of the form $\mathrm{A}(\alpha) \mathrm{B}(\beta)$, which is possible, provided that

$$
\begin{aligned}
\frac{\partial(\rho, z)}{\partial(\alpha, \beta)} & =f_{1}(\alpha)+f_{2}(\beta), \ldots . \\
\frac{1}{\rho} \frac{\partial \rho}{\partial \alpha} & =\text { function of } \alpha \text { only, } \\
\frac{1}{\rho} \frac{\partial \rho}{\partial \beta} & =\text { function of } \beta \text { only. }
\end{aligned}
$$


The two latter conditions may be combined to

$$
\rho=f_{3}(\alpha) \cdot f_{4}(\beta) \text {. . . . . . }
$$

The general solution of (11) has been given by Prof. Michell *. For our present purpose, it is necessary to select those solutions which satisfy the further conditions (12).

Excluding the cases in which the conjugate transformation involves elliptic functions, it appears that the surfaces for which our problem may be solved are the sphere, spheroid, cone, paraboloid and hyperboloid of revolution. Since a cylinder is a particular form of surface of revolution, in which the bounding curve describes a circle of infinite radius, it is evident that the cases of the circular, elliptic, parabolic, and hyperbolic cylinders, as well as that of two infinite planes meeting at any angle, may also be solved. It is found that the anchor-ring, a surface of considerable physical interest, does not admit of such a solution.

We proceed to discuss the vibrations between two surfaces of revolution of spherical and spheroidal shape. When they are perfectly conducting, the surface conditions are that the resultant magnetic induction and electric force at the surface are tangential and normal respectively. There are thus two distinct classes of vibrations, corresponding to $\phi=0$, and $\frac{\partial \phi}{\partial \alpha}=0$, if $\alpha=$ const. is one of the surfaces.

\section{Vibrations of Concentric Spheres.}

The periods of this simple system have been obtained by Macdonald $t$, as the roots of a complicated transcendental equation. If $(r \theta \omega)$ are spherical polar coordinates referred to the centre of the spheres,

$$
\begin{gathered}
z=r \cos \theta, \quad \rho=r \sin \theta, \\
\frac{\partial(\rho, z)}{\partial(\alpha, \beta)}=r^{2}, \quad \rho \frac{\partial}{\partial \alpha}\left(\frac{1}{\rho}\right)=-1, \quad \rho \frac{\partial}{\partial \beta}\left(\frac{1}{\rho}\right)=-\cot \theta,
\end{gathered}
$$

whence (10) becomes

$$
r^{2} \frac{\partial^{2} \phi}{\partial r^{2}}+\frac{\partial^{2} \phi}{\partial \theta^{2}}-\cot \theta \frac{\partial \phi}{\partial \theta}+k^{2} r^{2} \phi=0, \ldots .
$$

whose solution, finite for all inclinations $\theta$, is, if $\mu=\cos \theta$,

$$
\begin{gathered}
\phi=\sum_{n=0}^{\infty} r^{\frac{1}{2}} \sin ^{2} \theta \frac{d \mathrm{P}_{n}(\mu)}{d \mu}\left\{\mathrm{AJ}_{n+\frac{1}{2}}(k r)+\mathrm{B} \cdot J_{-n-\frac{1}{2}}(k r)\right\} . \\
* \text { Messenger of Math. vol. xix. } \\
+ \text { Loc. cit. }
\end{gathered}
$$


In the mode in which $\psi=0$, the only non-zero electrical components are $(x, y, c)$. Since $a=0$, the magnetic force at the surfaces is tangential, and the surface condition is merely $y=0$, or $\frac{\partial \phi}{\partial r}=0$. This leads to Macdonald's equation for the periods. The mode defined by $\psi$ leads to the other set of periods. The case $n=0$ makes all the components zero, and leads to no vibration.

The most interesting case is that of a single sphere. The most general components in a symmetrical oscillation inside the conductor are, if $c$ is the radius,

$$
\begin{aligned}
& x=p_{2} p_{3} \frac{\partial \phi}{\partial \theta}=\frac{\mathrm{A} n(n+1)}{r^{\frac{3}{2}}} \mathrm{~J}_{n+\frac{1}{2}}(k r) \cdot \mathrm{P}_{n}(\mu) \cos (k \mathrm{~V} t+\epsilon), \\
& y=-p_{1} p_{3} \frac{\partial \phi}{\partial(\log r)}=-\frac{\mathrm{A}}{r} \cdot \frac{d}{d r}\left(r^{\frac{2}{2} J_{n+\frac{1}{2}}}(k r)\right) \frac{d \mathrm{P}_{n}(\mu)}{d \mu} \cos (k \mathrm{~V} t+\epsilon), \\
& c=-\frac{\mathrm{A} k}{\overline{\mathrm{V}} r^{\frac{1}{2}}} \mathrm{~J}_{n+\frac{1}{2}}(k r) \cdot \sin ^{2} \theta \cdot \frac{d \mathrm{P}_{n}(\mu)}{d \mu} \sin (k \mathrm{~V} t+\epsilon),
\end{aligned}
$$

taken for all roots $k$ of

$$
\frac{d}{d c}\left\{c^{\frac{2}{2} J_{n+\frac{1}{3}}(k c)}\right\}=0, \ldots .
$$

together with

$$
\begin{aligned}
& a=\frac{\mathrm{B} n(n+1)}{r^{\frac{3}{2}}} \mathrm{~J}_{n+\frac{1}{2}}(k r) \mathrm{P}_{n}(\mu) \cos (k \mathrm{~V} t+\epsilon), \\
& b=\frac{\mathrm{B}}{r} \frac{d}{d r}\left(r^{\frac{1}{2}} \mathrm{~J}_{n+\frac{1}{2}}(k r)\right) \frac{d \mathrm{P}_{n}(\mu)}{d \mu} \cdot \cos (k \mathrm{~V} t+\epsilon), \\
& z=\frac{\mathrm{B} k}{\mathrm{~V} r^{\frac{1}{2}}} \mathrm{~J}_{n+\frac{1}{2}}(k r) \cdot \sin ^{2} \theta \cdot \frac{d \mathrm{P}_{n}(\mu)}{d \mu} \cdot \sin (k \mathrm{~V} t+\epsilon),
\end{aligned}
$$

taken for all roots $k$ of

$$
\mathbf{J}_{n+\frac{1}{2}}(k c)=0 . \quad . \quad . \quad . \quad .
$$

The gravest modes of the first and second systems (corresponding to $n=1$ in. each case) are given by the least roots of

$$
\left(k c-\frac{1}{k c}\right) \tan k c+1=0, \quad . \quad . \quad . \quad \text {. }
$$

and

$$
\tan k c=k c
$$

respectively. 
Vibrations of Conducting Surfaces of Revolution. $\quad 709$

Vibrations of Confocal Spheroids.

The proper transformation in the case of spheroids of ovary form is

which gives

$$
z+\iota \rho=c \cosh (\alpha+\iota \beta), \quad \cdot \quad \cdot \quad \cdot
$$

$$
\begin{gathered}
\rho=c \sinh \alpha \sin \beta=\frac{1}{p_{3}}, \\
\frac{\partial(\rho, z)}{\partial(\alpha, \beta)}=c^{2}\left(\cosh ^{2} \alpha-\cos ^{2} \beta\right) .
\end{gathered}
$$

Thus the characteristic equation (10) becomes

$$
\frac{\partial^{2} \phi}{\partial \alpha^{2}}+\frac{\partial^{2} \phi}{\partial \beta^{2}}-\operatorname{coth} \alpha \frac{\partial \phi}{\partial \beta}-\cot \beta \frac{\partial \phi}{\partial \beta}+k^{2} c^{2}\left(\cosh ^{2} \alpha-\cos ^{2} \beta\right) \phi=0 \text {. }
$$

To obtain a solution of the proper form, we may write, if $\lambda$ is some constant,

$$
\begin{aligned}
& \frac{\partial^{2} \phi}{\partial \alpha^{2}}-\operatorname{coth} \alpha \frac{\partial \phi}{\partial \alpha}+\left(k^{2} c^{2} \cosh { }^{2} \alpha-\lambda\right) \phi=0, \\
& \frac{\partial^{2} \phi}{\partial \beta^{2}}-\cot \beta \frac{\partial \phi}{\partial \beta}+\left(\lambda-k^{2} c^{2} \cos ^{2} \beta\right) \phi=0 .
\end{aligned}
$$

In the sphere, $\lambda=n .(n+1)$. Its proper values for the spheroid are to be determined so as to make $\phi$ recur when $\beta$ increases by $2 \pi$, and are of the form

$$
\lambda=n(n+1)+\epsilon_{n}(k c)^{2}+\delta_{n}(k c)^{4}+\ldots
$$

where $\epsilon_{n}, \delta_{n}, \ldots$ are functions of $n$ only.

Write $\phi=\sinh \alpha \cdot \sin \beta . A$. B, where $A$ is a function of $\alpha$ only, and $B$ of $\beta$ only.

We thus obtain

$$
\begin{aligned}
& \frac{d^{2} \mathrm{~A}}{d \alpha^{2}}+\operatorname{coth} \alpha \frac{d \mathrm{~A}}{d \alpha}+\mathrm{A}\left(k^{2} c^{2} \cosh ^{2} \alpha-\operatorname{cosech}^{2} \alpha-\lambda\right)=0 . \\
& \frac{d^{2} \mathrm{~B}}{d \beta^{2}}+\cot \beta \frac{d \mathrm{~B}}{d \beta}+\mathrm{B}\left(\lambda-k^{2} c^{2} \cos ^{2} \beta-\operatorname{cosec}^{2} \beta\right)=0 .
\end{aligned}
$$

These equations are included in a form considered by Niven * when discussing the conduction of heat in ellipsoids of revolution. His solution is unsuited to the present problem, but we may quote his value of $\lambda$. In the present case, it is found that

$$
\begin{aligned}
\lambda=n(n+1)+(k c)^{2} & \frac{2 n^{2}+2 n-3}{(2 n-1)(2 n+3)}-\frac{1}{2}(k c)^{4}\left\{\frac{n(n+1)(n+2)(n+3)}{(2 n+1)(2 n+3)^{3}(2 n+5)}\right. \\
& \left.-\frac{(n-2)(n-1)(n)(n+1)}{(2 n-3)(2 n-1)^{3}(2 n+1)}\right\}+\ldots . \quad \text {. }
\end{aligned}
$$

* Phil. Trans. 1880, p. 138. 
Thus when $n=0, \lambda=(k c)^{2}$ up to and including the seventh order. This is found to correspond to no vibration.

For the first three types of vibration, given by $n=1,2,3$, the values of $\lambda$ are

$$
\begin{aligned}
& \lambda_{1}=2+\frac{1}{5}(k c)^{2}-\frac{4}{7.5^{3}}(k c)^{4}+\frac{8}{21.5^{5}}(k c)^{6}-\ldots . \\
& \lambda_{2}=6+\frac{3}{7}(k c)^{2}-\frac{4}{3.7^{3}}(k c)^{4}+\frac{8}{33.7^{5}}(k c)^{6}-\ldots . \\
& \lambda_{3}=12+\frac{7}{15}(k c)^{2}+\frac{1064}{77.5^{3} \cdot 9^{2}}(k c)^{4}-\ldots \ldots .
\end{aligned}
$$

After the first term, these series converge very rapidly for values of $k c$ which are not very great, and more rapidly as $n$ increases. Now for the fundamental, and for the vibrations near to it in a sphere of radius $a, k a$ is not much greater than 1.or 2, and unless the spheroids differ greatly from the spherical shape, these series may be conveniently used in calculating the gravest vibrations of the doubly infinite system: For $c=a e$, where $a$ is the semi-axis major and $e$ the eccentricity.

Let $k c$ be denoted by $\epsilon$. We may pass from the spheroidal coordinates to spherical polars $(r, \theta, \omega)$ by making $\alpha$ great and $c$ small, so that $r=\frac{c}{2} e^{\alpha}, \quad \beta=\theta$.

It is known that the equation corresponding to (20) when $\alpha$ is infinite and $c$ zero, is satisfied by the form

$$
\phi=e^{\iota k r} f_{n}(\iota k r),
$$

where $f_{n}$ denotes a terminating polynomial in descending powers of $\iota k r$, which is useful for calculation for only moderate values of $k r$.

Write $\cosh \alpha=t$ in (20), and it becomes

$$
\left(t^{2}-1\right) \frac{d^{2} \phi}{d t^{2}}+\left(\dot{\epsilon}^{2} t^{2}-\lambda\right) \phi=0 . \quad . \quad .
$$

If in this transformed equation we write, following the method suggested by the case of the sphere,

we find that $r=0$.

$$
\phi=e^{\iota \epsilon t}\left(\frac{c_{r}}{(\iota \epsilon t)^{r}}+\frac{c_{r+1}}{(\iota \epsilon t)^{r+1}}+\ldots\right)
$$

Thus

$$
\phi=\epsilon^{\iota \epsilon t}\left(1+\frac{c_{1}}{\iota \epsilon t}+\frac{e^{\natural}}{(\iota \epsilon t)^{2}}+. .\right), . .
$$


where the series will not, in general, terminate. Denoting the series in brackets by $\mathrm{S}$, and substituting in the equation, we obtain, if $z=\iota \epsilon t$,

$$
\left(z^{2}+\epsilon^{2}\right)\left(\frac{d^{2} \mathbf{S}}{d z^{2}}+2 \frac{d \mathbf{S}}{d z}\right)+\left(\epsilon^{2}-\lambda\right) \mathrm{S}=0 .
$$

From this equation, the relations among successive coefficients become

$$
\begin{aligned}
2(m+1) c_{m+1} & =\left(\epsilon^{2}-\lambda+m . m+1\right) c_{m}-2(m-1) \epsilon^{2} c_{m-1} \\
\quad+(m-1)(m-2) \epsilon^{2} c_{m-2} & . \\
2 c_{1} & =\left(\epsilon^{2}-\lambda\right), \\
4 c_{2} & =\left(2+\epsilon^{2}-\lambda\right) c_{1}, \\
6 c_{3} & =\left(6+\epsilon^{2}-\lambda\right) c_{2}-2 \epsilon^{2} c_{1} .
\end{aligned}
$$

In the case of $n=0, \epsilon^{2}=\lambda$ to the seventh order. 'I'hus $c_{1}=0$, and therefore $c_{2}$ and successive coefficients are all zero. In fact, the solution in this case is, to this order,

$$
\phi=\mathrm{A} \cos (k c \cosh \alpha)+\mathrm{B} \sin (k c \cosh \alpha) \quad .
$$

so far as $\alpha$ is concerned.

The conjugate equation in $\beta$ is

$$
\frac{d^{2} \phi}{d \beta^{2}}-\cot \beta \frac{d \phi}{d \beta}+k^{2} c^{2} \sin ^{2} \beta=0,
$$

whose solution is accurately

$$
\phi=\mathrm{C} \cos (k c \cos \beta)+\mathrm{D} \sin (k c \cos \beta) . \quad .
$$

Now this is a function, which, even for great values of $k c$, is perfectly periodic in $\beta$, and is therefore a possible solution for $n=0$ for any value of $k c$. But there is only one such solution. Thus we infer without further calculation that for $n=0$, all the terms of Niven's series will vanish except $(k c)^{2}$, which is the accurate value of $\lambda_{0}$. Although this result is interesting from the point of view of several problems, it is not to be expected, from the analogy of the sphere, that it corresponds to any vibration in this case, and in fact, on writing down the values of the electric and magnetic forces by previous equations, it is found impossible to make them finite on the axis $(\beta=0)$ unless they are everywhere zero. We therefore pass to the case $n=1$, which leads to the 
true fundamental series of periods. When $n=1, \lambda=2+\frac{\epsilon^{2}}{5}$ to the second order, by (25),

$$
\begin{aligned}
& c_{1}=\left(\frac{2}{5} \epsilon^{2}-1\right) \\
& c^{2}=\frac{\epsilon^{2}}{5}\left(\frac{2}{5} \epsilon^{2}-1\right) \\
& c_{3}=\frac{\epsilon^{2}}{5} \text { to the same order }
\end{aligned}
$$

and $c_{4}$ and all higher coefficients commence with $\epsilon^{4}$.

Thus a solution for $n=1$, with small eccentricity, is

$$
\phi_{1}=e^{\imath \varkappa c \cosh \alpha}\left\{1-\frac{5-2 \epsilon^{2}}{5 \iota \epsilon \cosh \alpha}-\frac{\epsilon^{2}}{5(\iota \epsilon \cosh \alpha)^{2}}+\frac{\epsilon^{2}}{5(\iota \epsilon \cosh \alpha)^{3}}\right\}
$$

Another solution $\phi_{1}{ }^{\prime}$ is obtained by changing the sign of $\iota$.

When $n=2, \quad \lambda=6+\frac{3}{7} \epsilon^{2}$, by $(26)$,

$$
c_{1}=\frac{2}{7} \epsilon^{2}-3, \quad c_{2}=3-\frac{5}{7} \epsilon^{2}, \quad c_{3}=\frac{9}{7} \epsilon^{2}, \quad c_{4}=-\frac{9}{7} \epsilon^{2},
$$

and the higher coefficients commence with $\epsilon^{4}$.

Thus

$$
\begin{aligned}
\phi_{2}=e^{k i c \cosh \alpha}\{1 & \frac{21-2 \epsilon^{2}}{7 \iota \epsilon \cosh \alpha}+\frac{21-5 \epsilon^{2}}{7(\iota \epsilon \cosh \alpha)^{2}} \\
& \left.+\frac{9}{7} \cdot \frac{\epsilon^{2}}{(\iota \epsilon \cosh \alpha)^{3}}-\frac{9}{7} \cdot \frac{\epsilon^{2}}{(\iota \epsilon \cosh \alpha)^{4}}\right\} .
\end{aligned}
$$

and a solution $\phi_{\bar{\varepsilon}}{ }^{\prime}$ is obtained by changing the sign of $\iota$.

Similarly,

$$
\begin{array}{r}
\phi_{3}=e^{\iota k \cosh \alpha}\left\{1-\frac{90-4 \epsilon^{2}}{15 \iota \epsilon \cosh \alpha}+\frac{225-22 \epsilon^{2}}{15(\iota \epsilon \cosh \alpha)^{2}}-\frac{75-24 \epsilon^{2}}{5(\iota \epsilon \cosh \alpha)^{3}}\right. \\
\left.-\frac{10 \epsilon^{2}}{(\iota \epsilon \cosh \alpha)^{4}}+\frac{10 \epsilon^{2}}{(\iota \epsilon \cosh \alpha)^{5}}\right\} .
\end{array}
$$

Each succeeding function necessitates the addition of a new term. For vibrations between two confocal spheroids, a linear combination of any pair $\left(\phi, \phi^{\prime}\right)$ must be taken. Inside a single spheroid, we require a function finite at the centre. By comparison with the Bessel functions, it appears that if $n=1,3,5, \ldots$ the real part of $\phi$ must be taken, and if $n=2,4,6, \ldots$, the imaginary part. Thus the first three 
appropriate functions for internal vibrations are

$$
\begin{aligned}
\phi_{1}= & \left(1+\frac{1}{5} \operatorname{sech}^{2} \alpha\right) \cos (k c \cosh \alpha) \\
& -\left\{1-\frac{2}{5} k^{2} c^{2}+\frac{1}{5} \operatorname{sech}^{2} \alpha\right\} \frac{\sin (k c \cosh \alpha)}{k c \cosh \alpha}, \quad(36) \\
\phi_{2}= & \left(3-\frac{2}{7} k^{2} c^{2}+\frac{9}{7} \operatorname{sech}^{2} \alpha\right) \frac{\cos (k c \cosh \alpha)}{k c \cosh \alpha} \\
& +\left\{1-\frac{21-5 k^{2} c^{2}}{7 k^{2} c^{2} \cosh ^{2} \alpha}-\frac{9}{7} \frac{\operatorname{sech}^{4} \alpha}{k^{2} c^{2}}\right\} \sin (k c \cosh \alpha), \quad(37) \\
\phi_{3}= & \left\{1-\frac{75-22 k^{2} c^{2}}{15(k c \cosh \alpha)^{2}}-\frac{10 \operatorname{sech}^{4} \alpha}{k^{2} c^{2}}\right\} \cos (k c \cosh \alpha) \\
- & \left\{6-\frac{1}{15}(k c)^{2}-\frac{75-24 k^{2} c^{2}}{(5 k c \cosh \alpha)^{2}}-\frac{10 \operatorname{sech}^{3} c t}{k^{2} c^{2}}\right\} \frac{\sin (k c \cosh x)}{k c \cosh \alpha}
\end{aligned}
$$

In finding periods corresponding to any value of $n$, the equation in $\beta$ need not be solved, as it is known from the case of the sphere that the forces will be finite when $n$ is not zero We shall only examine the periods corresponding to $n=1$.

If $a$ is the major semi-axis, and $e$ the eccentricity, $c=a e$, $e=\operatorname{sech} \alpha$, where $\alpha$ relates to the boundary. The periods belonging to case 2 correspond to the surface condition $\phi_{1}=0$, when $n=0$.

Thus if $\sigma=k a$, the period equation becomes

$$
\frac{\tan \sigma}{\sigma}=1+\frac{2}{5} \sigma^{2} e^{2} \text {. . . . . . }
$$

the corresponding equation for the sphere being $\tan \sigma=\sigma$.

If $\delta$ be the positive correction for eccentricity of any root $\theta$ of $\tan \sigma=\sigma$,

$$
\begin{aligned}
\delta & =\frac{2}{5} e^{2} \theta^{2} / \frac{d}{d \theta}\left(\frac{\tan \theta}{\theta}\right) \\
& =\frac{2}{5} e^{2} \theta, . . \quad .
\end{aligned}
$$

writing $\tan \theta=\theta, \sec ^{2} \theta=1+\theta^{2}$.

The effect of a small eccentricity is therefore to increase $k a$ in the ratio $1+\frac{2}{5} e^{2}$, if a sphere become a spheroid with the same major axis. The wave-lengths are less in the spheroid in the ratio $1-\frac{2}{5} e^{2}$, and the correction is therefore Phil. Mag. S. 6. Vol. 11. No. 65. May 1906. 3 A 
very small. An eccentricity of $\frac{1}{2}$ reduces the wave-lengths in the ratio $\frac{9}{10}$ approxinately.

In case $1, n=1$, the period equation is $\frac{\partial \phi}{\partial \alpha}=0$.

This equation supplies the true fundamental of the system. With the previous notation, after some reduction, it becomes,

$$
\left(\frac{1}{\sigma}-\sigma\right) \tan \sigma=1+\frac{2 e^{2}}{5}\left(\frac{\sigma^{4}-3}{\sigma^{2}-1}\right)
$$

Let $\theta$ be a root of $\tan \sigma=\frac{\sigma}{1-\sigma^{2}}$, the period equation for the sphere, and $\delta$ be the correction for eccentricity. Then it may be at once shown that

$$
\begin{aligned}
& \delta=\frac{2}{5} e^{2} \cdot \frac{\theta^{4}-3}{\theta^{2}-1} / \frac{d}{d \theta}\left\{\frac{\tan \theta}{\theta}-\theta \tan \theta\right\} \\
& =-\frac{2}{5} e^{2} \cdot \frac{\theta^{4}-3}{\theta\left(\theta^{2}-2\right)} . \quad \text {. . . . . . . . . }
\end{aligned}
$$

For the higher roots, this becomes $\delta=-\frac{2}{5} e^{2} \theta$. The correction is therefore equal and opposite to that in case 2 , if the vibrations are not too near the fundamental. The fundamental of the sphere corresponds to a value of $\theta$ approximately equal to $2 \cdot 744$ or $7 \pi / 8$.

\section{The Oblate or Planetary Spheroid.}

The proper substitution in this case is

giving

$$
\rho+\iota z=c \cosh (\alpha+\iota \beta)
$$

$$
\begin{gathered}
\rho=c \cosh \alpha \cos \beta, \\
\frac{\partial(\rho, z)}{\partial(\alpha, \beta)}=c^{2}\left(\cosh ^{2} \alpha-\cos ^{2} \beta\right) ;
\end{gathered}
$$

and the equation for $\phi$ becomes

$$
\frac{\partial^{2} \phi}{\partial \alpha^{2}}+\frac{\partial^{2} \phi}{\partial \beta^{2}}-\tanh \alpha \frac{\partial \phi}{\partial \alpha}+\tan \beta \frac{\partial \phi}{\partial \beta}+\epsilon^{2}\left(\sinh ^{2} \alpha+\sin ^{2} \beta\right) \phi=0 .
$$

In this, write $\left(-\epsilon^{2}, \alpha-\frac{\ell \pi}{2}, \beta-\frac{\pi}{2}\right)$ for $\left(\epsilon^{2}, \alpha, \beta\right)$ respectively, and it becomes identical with (20). Thus to apply the previous results to an oblate spheroid, it is only necessary to make these three substitutions in them. In previous investigations, $\cosh \alpha$ becomes $\iota \sinh \alpha$; $\sinh \alpha, \iota \cosh \alpha$; $\cos \beta,-\sin \beta ; \sin \beta, \cos \beta ;$ and $k c \cosh \alpha, k c \sinh \alpha$. The 
Vibrations of Conducting Surfaces of Revolution.

major axis of the previous spheroid is to be replaced by the minor axis of the new one. The sign of $e^{2}$ is to be changed wherever it occurs. Thus the corrections of the fundamental series of periods for eccentricity are equal and opposite to those of the other type of spheroids. The detailed theory for the oblate spheroids will not be given, as the previous formulæ may be at once transformed properly.

\section{7he Higher Periods of Spheres and Spheroids.}

In cases 1 and 2 of the sphere, when $n$ is small, and the higher periods are being treated, the period equations become, adopting the ordinary asymptotic expansion of Bessel functions,

$$
\left.\begin{array}{l}
\cos \\
\sin
\end{array}\right\}\left(k a-(n+1) \frac{\pi}{2}\right)=0 . \quad . \quad . .
$$

Thus if $m$ be a large integer, a possible wave-length for a sphere of radius $a$ is $\frac{4 a}{m}$ approximately.

When $n$ is appreciable, the ordinary expansion of Bessel functions ceases to apply. But it was shown by the author* that the proper expansion in this case is

$\mathrm{J}_{n+\frac{1}{2}}(k r)=\sqrt{\frac{2}{\pi}} \frac{\cos \left\{\sqrt{k^{2} r^{2}-n \cdot n+1}-\overline{n+\frac{1}{2}} \cos ^{-1} \frac{(n \cdot n+1)^{\frac{\pi}{2}}}{k r}-\frac{\pi}{4}\right\}}{\left(k^{2} r^{2}-n \cdot n+1\right)^{\frac{1}{4}}}$,

and the two period equations become

$$
\begin{aligned}
& \sqrt{k^{2} a^{2}-n \cdot n+1}-\left(n+\frac{1}{2}\right) \cos ^{-1} \sqrt{\frac{n \cdot n+1}{k^{2} c^{2}}}=(4 s+3) \frac{\pi}{4} \quad(s \text { large }) \text {. } \\
& \quad \text { and }
\end{aligned}
$$

$$
\begin{aligned}
\tan \left\{\sqrt{k^{2} a^{2}-n \cdot n+1}\right. & \left.-\left(n+\frac{1}{2}\right) \cos ^{-1} \sqrt{\frac{n \cdot n+1}{k^{2} a^{2}}}-\frac{\pi}{4}\right\} \\
& =-\frac{n \cdot n+1}{2(k a)^{\frac{2}{2}}\left(k^{2} a^{2}-n \cdot n+1\right)^{\frac{7}{4}}} .
\end{aligned}
$$

These two formula will supply the correction necessary to the periods deduced from the ordinary expansion, when $n$ becomes appreciable, if expanded in powers of $\frac{n}{k a}$.

However great $n$ may be, these equations will give the higher modes of the series defined by $n$, and will do so more accurately as $n$ increases, it the higher roots are taken. But

$$
\text { * Phil. Mag. Feb. 1906, p. } 195 .
$$


these high frequencies are less interesting physically. The fundamentals of series defined by large values of $n$ are themselves high modes, in comparison with the fundamental of the system.

We now prareed to treat the higher vibrations of spheroids corresponding to any values of $n$. The equation in $\beta$ is

$$
\frac{d^{2} \phi}{d \beta^{2}}-\cot \beta \frac{d \phi}{d \beta}+\left(\lambda-k^{2} c^{2} \cos ^{2} \beta\right) \phi=0 \text {. }
$$

Adopting the substitution *

we obtain

$$
\phi=e^{l k c \omega} \cdot \psi, . . \quad . \quad . \quad .
$$

$$
\left(\psi^{\prime \prime}-\cot \beta \psi^{\prime}\right)-\imath k c\left(2 \omega^{\prime} \psi^{\prime}+\psi \omega^{\prime \prime}-\omega^{\prime} \psi \cot \beta\right)
$$

$$
+\psi k^{2} c^{2}\left(\frac{\lambda}{\epsilon^{2}}-\cos ^{2} \beta-\omega^{\prime 2}\right)=0 .
$$

Neglecting the first bracket, and equating the brackets of different orders in $k c$ to zero,

$$
\begin{gathered}
\omega^{\prime}=\sqrt{\frac{\lambda}{\epsilon^{2}}-\cos ^{2} \beta,} \\
\frac{2 \psi^{\prime}}{\psi}+\frac{\omega^{\prime \prime}}{\omega^{\prime}}-\cot \beta=0 .
\end{gathered}
$$

Thus if $\omega=\int \beta \sqrt{\frac{\lambda}{\epsilon^{2}}-\cos ^{2} \beta} d \beta$, the solution becomes

$$
\phi=\frac{A \cos k c \omega+B \sin k c \omega}{\left(\frac{\lambda}{\epsilon^{2}}-\cos ^{2} \beta\right)^{\frac{1}{4}}} \cdot \sqrt{\sin \beta}, \quad \text {. }
$$

except close to the axis of the spheroids.

From this we may obtain the limiting value of $\lambda$ when $n$, although possibly large, is small in comparison with $\epsilon$ or $k$. The denominator of $\phi$ as written has a period $2 \pi$, and therefore $\phi$ has, if $\omega$ has. This occurs if $\lambda=\epsilon^{2}$, if the solution remains finite and uniform at $\beta=0$. Now writing

$$
\lambda=\epsilon^{2}+n \cdot n+1,
$$

and putting $\cos \beta=\mu$, we obtain

$$
\left(1-\mu^{2}\right) \frac{d^{2} \phi}{d \mu^{2}}+\left(n . n+1+\epsilon^{2}-\epsilon^{2} \mu^{2}\right) \phi=0, . \quad .
$$

This is satisfied by a convergent series in rising powers of $\mu$, even in $\mu$ if $n$ is odd, and odd if $n$ is even. By putting $\mu= \pm 1-\eta$, the solution is finite at $\mu= \pm 1$, and therefore on the axis of the spheroids. Thus a solution of the required

* Webb, Proc. Roy. Soc. 1904, p. 315. 
form exists. The asymptotic expansion therefore represents (except on the axis) a function finite at all points, if it satisfies the odd and even conditions.

Thus $B=0$ if $n$ is odd, and $A=0$ if $n$ is even. The continuation of the function on the axis is not given by the expansion.

When $k e$ is great in comparison with $n, \lambda$ therefore becomes $(k c)^{2}$, and the equation in $\alpha$ for $\phi$ then has the simple solution given by (31). Thus

$$
\phi=\mathrm{C} \cos (k c \cosh \alpha)+\mathrm{D} \sin (k c \cosh \alpha)
$$

This is, of course, another asymptotic expansion, suitable for the space between two spheroids, and representing the proper function except at the origin, where its continuation must be differently expressed. $\mathrm{D}=0$ if $n$ is odd, and $\mathrm{C}=0$ if $n$ is even, if we are treating the interior of a spheroid. When the eccentricity is very small, the $\beta$ factor becomes incorrect, and must be replaced by $\left(1-\mu^{2}\right) \frac{d \mathrm{P}_{n}(\mu)}{d \mu}$ where $\mu=\cos \beta$. But the $\alpha$ factor remains correct, for $c \cosh \alpha$ becomes $r$ when the eccentricity is small, and the $\alpha$ factor becomes the ordinary expansion of

$$
r \cdot \frac{1}{2}\left\{\mathrm{~J}_{n+\frac{1}{2}}(k r)+\nu \mathrm{J}_{-n-\frac{1}{2}}(k r)\right\}
$$

which is the true factor for a sphere (cf. supra).

If $n$ is retained, the more accurate expression for $\phi$ becomes

$$
\phi=\frac{\mathrm{A} \sqrt{\sinh \alpha \sin \beta} \cos \left(\epsilon \omega^{\prime}-\epsilon_{1}\right)_{\sin }^{\cos }}{\left(\epsilon^{2} \sinh ^{2} \alpha-n \cdot n+1\right)^{\frac{1}{4}}\left(n \cdot n+1+\epsilon^{2} \sin ^{2} \beta\right)^{\frac{1}{4}}} \cdot \cos k \mathrm{~V} t,
$$

where $\epsilon_{1}, \epsilon_{2}$ are arbitrary constants, and

$$
\begin{aligned}
& \epsilon \omega^{\prime}=\int^{\alpha}\left(\epsilon^{2} \sinh ^{2} \alpha-n . n+1\right)^{\frac{1}{2}} d a, \ldots \\
& \epsilon \omega=\int^{\beta}\left(n . n+1+\epsilon^{2} \sin ^{2} \beta\right)^{\frac{1}{2}} d \beta, \ldots
\end{aligned}
$$

This is proved for the $\alpha$ factor just as in (50).

If $n$ be neglected, the high periods between two spheroids $\left(\alpha_{1}, \alpha_{2}\right)$ in Case 2 are given by

$$
A \cos \left(k c \cosh \alpha_{1},{ }_{2}\right)+\beta \sin \left(k c \cosh \alpha_{1},{ }_{2}\right)=0,
$$

whence

$$
k\left(a_{1}-a_{2}\right)=s \pi \text {. }
$$

In Case 1,

$$
k\left(a_{1}-a_{2}\right)=(2 s+1) \frac{\pi}{2}, .
$$

where $a_{1}, a_{2}$ are the major axes. These are replaced by the minor axes in the case of oblate spheroids. 
The high periods of a single spheroid are given by

$$
\left.\begin{array}{l}
\cos \\
\sin
\end{array}\right\} \int^{\alpha}\left(k^{2} c^{2} \cosh ^{2} \alpha-n \cdot n+1\right)^{\frac{1}{2}} d \alpha=0, \quad .
$$

$$
\begin{aligned}
& \frac{d}{d \alpha}\left\{\frac{\sqrt{\sinh \alpha}}{\left(k^{2} c^{2} \cosh ^{2} \alpha-n \cdot n+1\right)^{\frac{1}{4}}}\right. \\
& \left.\times \begin{array}{c}
\cos \\
\sin
\end{array}\right\}\left\{\left(k^{2} c^{2} \cosh ^{2} \alpha-n \cdot n+1\right)^{\frac{1}{2} d x}\right\}=0,
\end{aligned}
$$

where the upper or lower functions are taken according as $n$ is odd or even. These give the high vibrations of the singly infinite system defined by $n$.

The investigation given in a previous paper* for the elliptic cylinders becomes, if stated in full detail, exactly similar to the present. The $\beta$ factor is restricted in a similar way, but its adjustment for uniformity on the axis is incorrectly given. The true value, in the notation there employed, oscillates between $\mu^{-\frac{1}{4}} \cosh k \sqrt{b^{2}+\mu}$ and $\mu^{-\frac{1}{4}} \sinh k \sqrt{b^{2}+\mu}$ according to the value of $n$. The equation there numbered (51) ought to be written

$$
\left.\frac{d}{d \lambda}\left\{\frac{\cos }{\sin }\right\}\left(k \sqrt{b^{2}+\lambda}-\frac{\pi}{4}\right)\right\}=0, \quad . \quad .
$$

according to the value of $n$. The $\frac{\pi}{4}$ is determined most readily from the corresponding Bessel function.

Series of Periods corresponding to High Values of $\mathrm{n}$.

The solution may be expressed approximately in the general case when $n$ is large, even though $k c$ may not be so large that the value of $\lambda$ is $(k c)^{2}$. Writing $\lambda=n . n+1+\lambda^{\prime}$, or, when $n$ is large, $\lambda=n^{2}+\lambda^{\prime}$, the asymptotic expansion of the equations for $\phi$ when $n$ is large, found just as in the case when $k c$ is large, lead to

$$
\phi=\frac{A \cos \left(\omega_{1}+\epsilon_{1}\right) \cos \left(\omega_{2}+\epsilon_{2}\right) \cos k V t}{\left(\omega_{1}^{\prime} \omega_{2}^{\prime}\right)^{\frac{1}{2}}} \sqrt{\sinh x \sin \bar{\beta}} .
$$

where

$$
\begin{aligned}
& \omega_{1}^{\prime}=\frac{d \omega_{1}}{d \alpha}=\left(\epsilon^{2} \cosh ^{2} \alpha-\lambda^{\prime}-n^{2}\right)^{\frac{1}{2}}, \ldots . . \\
& \omega_{2}^{\prime}=\frac{d \omega_{2}}{d \alpha}=\left(n^{2}+\lambda^{\prime}-\epsilon^{2} \cos ^{2} \beta\right)^{\frac{1}{2}}, \quad . \quad . \quad . \\
& \quad * \text { Loc. cit. }
\end{aligned}
$$


and the value of $\phi$ may be expressed in terms of elliptic functions, as was previously done for the elliptic cylinder (loc. cit.). It may be noted that $\omega_{1}$ and $\omega_{2}$ are both real for all roots $\epsilon$. This formula holds for all values of $\beta$ if $n$ is not very small compared with $k c$, and does not fail on the axis.

It is convenient to note here that in the case of the elliptic cylinder corresponding to the above, $n^{2}$ should be $-n^{2}$, and the quantity $\theta$ there employed must be retained in full as a series in powers of $(k c)^{2}$. The series in two dimensions, given by Mathieu in connexion with the vibrations of an elliptic membrane, is,

$$
\theta=n^{2}+\frac{(k b)^{4}}{2\left(n^{2}-1\right)}+\frac{(k b)^{8} \cdot 5 n^{2}+7}{32\left(n^{2}-1\right)^{3}\left(n^{2}-4\right)}+\ldots, \quad .
$$

corresponding to

$$
\frac{d^{2} \mathrm{M}}{d \eta^{2}}+\left(\theta-2 k^{2} b^{2} \cos 2 \eta\right) \mathrm{M}=0 . \quad . \quad . \quad .
$$

The period equations for the vibrations between two spheroids, corresponding to large values of $n$, may be written down at once from $(60)$.

Selecting the function corresponding to the internal Bessel function of the sphere, by writing $c=\lambda^{\prime}=0$, it appears that $\epsilon_{1}$ in $(60)$ is $\frac{\pi}{4}$ for the single spheroid. Thus for the higher series, the period equations for a single spheroid are

and

$$
\int_{0}^{a}\left(\epsilon^{2} \cosh ^{2} \alpha-\lambda^{\prime}-n^{2}\right)^{\frac{1}{2}}=(4 s+1) \frac{\pi}{4}, \ldots .
$$

$$
\frac{d}{d \alpha}\left\{\left(\frac{\sinh \alpha}{\omega_{1}^{\prime}}\right)^{\frac{1}{2}} \cos \left(\omega_{1}-\frac{\pi}{4}\right)\right\}=0 . \quad .
$$

In the case of the elliptic eylinder, there is only one such equation, namely

$$
\frac{d}{d \alpha} \cdot \frac{\cos \left(\omega_{1}-\frac{\pi}{4}\right)}{\left(\omega_{1}^{\prime}\right)^{\frac{1}{2}}}=0, \quad \text {. . . . . }
$$

the value of $\lambda^{\prime}$ being given by (63) above.

\section{Vibrations in the External Space.}

The vibrations in the space external to a single conductor have, in general, a large modulus of decay, and cannot be maintained for a great space of time. The form of $\phi$ suitable for the space, employing imaginary quantities, must 
contain an exponential factor of negative imaginary argument. By this consideration, all previous formulæ for periods may be at once transformed into those proper to the external space.

In the case of the sphere, when $n=1$, the proper form of $\phi$ is, so far as concerns $r$,

$$
\phi=\mathrm{A} e^{-\imath k r}\left(1+\frac{1}{\iota k r}\right) . \text {. . . . . }
$$

In the second type of vibration, $\phi=0$ when $r=a$.

Thus

$$
1+\frac{1}{i k a}=0 \text {, }
$$

and

$$
e^{2 l i \mathrm{~V} t}=e^{-\frac{\mathrm{v} t}{u}}
$$

so that there is really no vibration at all, but a simple decay at a very rapid rate, on account of the large value of $V$. In the first type, $\frac{\partial \dot{\phi}}{\partial r}=0$, when $r=a$, leading to

$$
1+i k-k^{2} a^{2}=0,
$$

or

$$
k a=\frac{1}{2}(\iota+\sqrt{3}) \text {. }
$$

The time factor becomes

$$
e^{-\frac{\mathrm{v} t}{2 a}} \cdot e^{i \sqrt{3} \frac{\mathrm{v} t}{2 u}},
$$

and therefore the real value of $k a$ is $\frac{\sqrt{3}}{2}$, but there is, in addition, a rapid decay of the vectors at half the rate of the previous type. In the case of the prolate spheroid, when $n=1$, by changing the sign of $\iota$ in (33),

$$
\phi=e^{-\imath \epsilon \cosh \alpha}\left\{1+\frac{1-\frac{2}{5} \epsilon^{2}}{\iota \epsilon \cosh \alpha}-\frac{\epsilon^{2}}{5(\iota \epsilon \cosh \alpha)^{2}}-\frac{\epsilon^{2}}{5(\iota \epsilon \cosh \alpha)^{3}}\right\}
$$

Taking the second type of vibration, corresponding to $\phi=0$ at $c \cosh \alpha=a$, where $a$ is the major semiaxis, and putting $\cosh \alpha=\frac{1}{e}, \quad k a=\sigma$,

or neglecting $e^{4}$,

$$
1+\frac{1-\frac{2}{5} \sigma^{2} e^{2}}{\iota \sigma}+\frac{\sigma^{2} e^{2}}{5 \sigma^{2}}+\frac{\sigma^{2} e^{2}}{\tilde{\jmath} \iota \sigma^{3}}=0,
$$

$$
1+\iota \sigma-\frac{2}{5} \sigma^{2} e^{2}=0 .
$$


If $\sigma=\imath+\delta$, we find

$$
\delta=\frac{2}{5} e^{2} \iota . \quad \cdot \quad \cdot \quad \cdot \quad .
$$

The vectors therefore decay more rapidly than in the case of the sphere, the rate being increased in the ratio $\left(1+\frac{2}{5} t^{2}\right)$. For the other type, the period equation reduces to

$$
\iota\left(\frac{1-\sigma^{2}}{\sigma}\right)=1+\frac{2}{5} e^{2}\left(\begin{array}{c}
\sigma^{4}-3 \\
\sigma^{2}-1
\end{array}\right) .
$$

If

$$
\begin{aligned}
& \sigma=\frac{1}{2}(\iota+\sqrt{3})+\delta, \\
& \delta=(\iota \sqrt{ } 3-7) \frac{e^{2}}{5 \sqrt{ } 3} \cdot . . .
\end{aligned}
$$

Thus the new rate of decay is $\frac{\mathrm{V}}{a}\left(\frac{1}{2}+\frac{e^{\mathrm{z}}}{5}\right)$, and the decay is again more rapid than in the ease of the sphere. The real value of $k a$ becomes $\frac{\sqrt{3}}{2}-\frac{7 e^{2}}{5 \sqrt{3}}$, and is decreased. For the oblate spheroid, the sign of $e^{2}$ must be altered. A particular case of the oblate spheroid is the circular disk, which is worthy of separate treatment.

All formulæ above for the high periods may be transformed as shown, so as to be suitable for the external space.

\section{Vibrations of other Surfaces of Revolution.}

The cone is best treated by spherical polar coordinates. The transformation appropriate to the paraboloid is

$$
\rho+\iota z=(\alpha+\iota \beta)^{2} . \text {. . . . }
$$

In two dimensions, this will solve the problem of the parabolic cylinder. The appropriate functions for this case have been treated by Whittaker *.

Hyperboloids of revolution form the conjugate case to spheroids, but are of little physical interest. The hyperbolic cylinder may be solved in the same manner. 